\title{
INFINITE RANK BUTLER GROUPS
}

\author{
MANFRED DUGAS AND K. M. RANGASWAMY
}

\begin{abstract}
A torsion-free abelian group $G$ is said to be a Butler group if $\operatorname{Bext}(G, T)$ $=0$ for all torsion groups $T$. It is shown that Butler groups of finite rank satisfy what we call the torsion extension property (T.E.P.). A crucial result is that a countable Butler group $G$ satisfies the T.E.P. over a pure subgroup $H$ if and only if $H$ is decent in $G$ in the sense of Albrecht and Hill. A subclass of the Butler groups are the so-called $B_{2}$-groups. An important question left open by Arnold, Bican, Salce, and others is whether every Butler group is a $B_{2}$-group. We show under $(V=L)$ that this is indeed the case for Butler groups of rank $\aleph_{1}$. On the other hand it is shown that, under ZFC, it is undecidable whether a group $B$ for which $\operatorname{Bext}(B, T)=0$ for all countable torsion groups $T$ is indeed a $B_{2}$-group.
\end{abstract}

1. Introduction. The class of completely decomposable torsion-free abelian groups is one of the few classes of abelian groups that can be completely determined by isomorphism invariants, as was shown by R. Baer [5]. In 1965 M. C. R. Butler [10] initiated the study of the pure subgroups of finite rank completely decomposable groups and in recent years this class of groups has been studied in greater detail by D. Arnold [2, 4], D. Arnold and C. Vinsonhaler [3], L. Bican [6, 7], L. Bican and L. Salce [8], and others. These finite rank torsion-free abelian groups have been called Butler groups and are shown to have several interesting characterizing properties including the following striking result by L. Bican [6]: A finite rank torsion-free abelian group $G$ is a Butler group if and only if $\operatorname{Bext}(G, T)=0$ for all torsion groups $T$. Here Bext denotes the subfunctor of the functor Ext defined by the proper class of balanced exact sequences [13].

In their attempt to generalize the properties of (finite rank) Butler groups to infinite rank groups, Bican and Salce [8], Bican, Salce, and Stepan [9], and Arnold [4] were led to the following three classes of abelian groups:

$\mathscr{B}_{1}$ The class of torsion-free abelian groups $G$ for which $\operatorname{Bext}(G, T)=0$ for all torsion groups $T$

Received by the editors January 5, 1987. Presented at The 93rd Annual Meeting of the American Mathematical Society at San Antonio, Texas, January 21, 1987.

1980 Mathematics Subject Classification (1985 Revision). Primary 20K20; Secondary 20K35, 20K40.

Key words and phrases. Torsion-free abelian groups, Butler groups, pure subgroups.

Supported by a research grant from the College of Engineering and Applied Science, University of Colorado, Colorado Springs. 
$\mathscr{B}_{2}$ The class of torsion-free abelian groups $G$ which have a $B$-filtration, that is, $G$ is the union of a smooth chain

$$
0=G_{0} \subseteq G_{1} \subseteq \cdots \subseteq G_{\alpha} \subseteq \cdots
$$

of pure subgroups $G_{\alpha}$ such that, for each $\alpha, G_{\alpha+1}=G_{\alpha}+B_{\alpha}$ where $B_{\alpha}$ is a finite rank Butler group.

$\mathscr{B}_{3}$ The class of torsion-free abelian groups $G$ such that $G$ is locally completely decomposable and that each finite rank pure subgroup of $G$ is a Butler group.

Bican and Salce [8] and Bican, Salce, and Stepan [9] showed that these three classes coincide for countable groups and that $\mathscr{B}_{2}$ is a subclass of both $\mathscr{B}_{1}$ and $\mathscr{B}_{3}$. Are these three classes in general different or not? This question was raised by $\mathrm{D}$. Arnold [4], left open by Bican and Salce [8], and also mentioned by Albrecht and Hill [1]. It is debatable which one of these three classes of groups should be called the infinite rank Butler groups. Albrecht and Hill [1] show that a torsion-free group $G$ has a $B$-filtration if and only if $G$ satisfies axiom 3 with respect to decent subgroups. Here a subgroup $S$ of $G$ is said to be decent in $G$ if for any finite subset $X$ of $G$ there is a finite rank Butler group $B$ such that $S+B$ is pure in $G$ and contains $X$.

In conformity with the terminology of [8] and also because of the homological nature of this property, we call an arbitrary rank torsion-free abelian group $G$ a Butler group if $\operatorname{Bext}(G, T)=0$ for all torsion groups $T$. We refer to members of the class $\mathscr{B}_{2}$ as $B_{2}$-groups. As a first step in our investigation of infinite rank Butler groups, we prove that a finite rank Butler group $G$ has the following, perhaps interesting, torsion extension property (T.E.P.): Every pure exact sequence $0 \rightarrow K$ $\rightarrow G \rightarrow H \rightarrow 0$ induces, for any torsion group $T$, the exact sequence

$$
0 \rightarrow \operatorname{Hom}(H, T) \rightarrow \operatorname{Hom}(G, T) \rightarrow \operatorname{Hom}(K, T) \rightarrow 0 .
$$

Interestingly the infinite rank Butler groups do not seem to have the T.E.P. A result (Theorem 7) which is crucial in our later investigation says that if $B$ is a Butler group of countable infinite rank, then $B$ satisfies the T.E.P. over a pure subgroup $A$, if and only if $A$ is decent in $B$.

Using this and employing Gödel's axiom of constructibility $(V=L)$ we are able to show that a Butler group $B$ of rank $\aleph_{1}$ in which every finite rank pure subgroup is Butler admits a $B$-filtration, i.e., is a $B_{2}$-group. Actually we prove something stronger: Instead of $B$ being a Butler group we only need $\operatorname{Bext}(B, T)=0$ for any countable, $\Sigma$-cyclic torsion group. This shows that under $V=L$, countable torsion groups are enough to test whether a torsion-free group is Butler.

This compares with P. Eklof's analysis of the splitting problem of mixed groups [12]. The Baer splitting problem, solved by Griffith [14], becomes undecidable in standard ZFC set theory if one restricts the class of torsion groups to the countable ones. The same happens here for the splitting of balanced extensions. P. Eklof [11] showed that Martin's axiom and the negation of the continuum hypothesis imply that $\operatorname{Ext}(A, C)=0$ for any strongly $\boldsymbol{\aleph}_{1}$-free group $A$ and any countable group $C$. This readily leads to examples of groups $B$ such that $\operatorname{Bext}(B, T)=0$ for all countable torsion groups $T$ but $B$ is neither in $\mathscr{B}_{2}$ nor in $\mathscr{B}_{3}$. 
2. Preliminaries. All the groups that we consider here are abelian and unless otherwise specified, they are additively written torsion-free abelian groups. We generally follow the notation and terminology of [13]. For each prime $p, \mathbf{Z}_{p}$ denotes the group of rational numbers whose denominators are prime to $p$. For any group $G$ and a nonempty set of primes $S, G_{S}$ denotes the localization $\mathbf{Z}_{S} \otimes G$, where $\mathbf{Z}_{S}=\bigcap\left\{\mathbf{Z}_{p}: p \in S\right\}$. In particular, $G_{\{p\}}$ is denoted by $G_{p}$ for any prime $p$. If $G$ is a torsin group, then $G_{p}$ is simply the $p$-component of $G$. A height is a sequence $h=\left(h_{p}\right)$, indexed by the set $\mathbf{P}$ of all primes $p$ such that $h_{p}$ is an ordinal or the symbol $\infty$ for each $p$. If $G$ is an abelian group and $x \in G$, then $|x|=\left(h_{p}^{G}(x)\right)$ is a height where $h_{p}^{G}(x)$ is a $p$-height of $x$ in $G$. If $h=\left(h_{p}\right)$ is a height and $n$ an integer, then $n h$ is the height whose $p$ th entry is $h_{p}+h_{p}^{\mathbf{Z}}(n)$. Two heights $h$ and $h^{\prime}$ are equivalent, $h \sim h^{\prime}$ if there are integers $m$ and $n$ such that $m h=n h^{\prime}$. The relation $\sim$ is an equivalence relation on heights and the equivalence classes of heights are called types. For $x \in G, \tau(x)$ denotes the type of $x$ being the equivalence class of $|x|$. For any height $h, G(h)=\{x \in G:|x| \geqslant h\}$. An exact sequence $0 \rightarrow A \rightarrow B \rightarrow$ $C \rightarrow 0$ of abelian groups is balanced if, for each height $h$, the induced sequence $0 \rightarrow A(h) \rightarrow B(h) \rightarrow C(h) \rightarrow 0$ is exact. A useful result is that a balanced exact sequence $0 \rightarrow A \rightarrow B \rightarrow C \rightarrow 0$ splits if $C$ is a completely decomposable group.

If $S$ is a subset of a torsion-free group $G$, then $\langle S\rangle_{*}$ denotes the pure subgroup generated by $S$. A subgroup $H$ of $G$ is said to be a generalized regular subgroup of $G$ if $G / H$ is torsion and for each rank one pure subgroup $R$ of $G,(R /(R \cap H))_{p}=0$ for almost all primes $p$. A torsion-free group $G$ is said to be locally completely decomposable if, for each prime $p, G_{p}$ is completely decomposable. If $f: A \rightarrow B$ is a map and $S \subseteq A$, then $f \mid S$ denotes the restriction of $f$ to $S$. If $x \in T$, where $T$ is a torsion group, and $p$ is a prime, then $x_{p}$ denotes the $p$-component of $x$ in $T$.

3. The torsion extension property. In this section we show that the finite rank Butler groups satisfy a special property that may be of independent interest. This property turns out to be vital in our investigation of the infinite rank Butler groups in the following sections.

It is known (see [13]) that a pure subgroup of a homogeneous completely decomposable group $G$ of finite rank is a direct summand of $G$. Our first lemma extends this to the case when the typeset of $G$ is linearly ordered.

Lemma 1. Let $G$ be a finite rank completely decomposable group whose typeset is linearly ordered. Then every pure subgroup $A$ of $G$ is a quasi-summand; that is, there is a (completely decomposable) subgroup $G^{\prime}$ with $G^{\prime}=A \oplus X$ and $G / G^{\prime}$ finite.

Proof. The proof is by induction on the rank $k$ of $A$. Suppose $k=1$, say, and $A=\langle a\rangle_{*}$. Let $G=\oplus_{i=1}^{n}\left\langle x_{i}\right\rangle_{*}$ with $\left|x_{1}\right| \leqslant\left|x_{2}\right| \leqslant \cdots \leqslant\left|x_{n}\right|$. Without loss of generality we may assume that $a$ has a nonzero component in $\left\langle x_{1}\right\rangle_{*}$. Then clearly $\tau(a)=\tau\left(x_{1}\right)$. Let $G^{\prime}=\langle a\rangle_{*} \oplus \sum_{i=2}^{n}\left\langle x_{i}\right\rangle_{*}$. If $\sigma: G \rightarrow\left\langle x_{1}\right\rangle_{*}$ is the coordinate projection, then $L=\sigma\left(\langle a\rangle_{*}\right)$ has the same type as $\left\langle x_{1}\right\rangle_{*}$ and so $G / G^{\prime} \cong\left\langle x_{1}\right\rangle_{*} / L$ is finite.

Suppose rank $A=k>1$ and $B$ is a pure subgroup of $A$ of rank $k-1$. By the induction hypothesis, there is a completely decomposable subgroup $H$ of $G$ with 
$H=B \oplus C$ and $G / H$ finite. Then $A \cap H=B \oplus(A \cap C)$ and since $A / B$ has rank one, $A \cap C$ is a rank one pure subgroup of $C$. Since $H$ has the same typeset as $G$, the typeset of $C$ is linearly ordered. Hence, as in the preceding paragraph, we have a completely decomposable subgroup $C^{\prime}$ of $C$ with $C^{\prime}=(A \cap C) \oplus D$ and $C / C^{\prime}$ finite. Then $G^{\prime}=A+D=A \oplus D$ has finite index in $G$ and, by Butler [10], $G^{\prime}$ is completely decomposable.

Lemma 1 enables us to prove the following interesting property of Butler groups of finite rank.

THEOREM 2. Let $A$ be a pure subgroup of a finite rank Butler group $B$. Then every homomorphism $f: A \rightarrow T$, where $T$ is any torsion group, extends to a homomorphism $g: B \rightarrow T$.

Proof. By Bican [7], there is a partition $\mathbf{P}=\bigcup_{i=1}^{n} S_{i}$ of the set $\mathbf{P}$ of all primes such that, for each $i=1, \ldots, n$, the localization $B_{S_{i}}$ is a completely decomposable group whose typeset is linearly ordered. By Lemma 1 , there is a completely decomposable subgroup $C_{i}$ of $B_{S_{i}}$ with finite index $n_{i}$ such that $C_{i}=A_{S_{i}} \oplus D_{i}$. Let $P_{i}$ be the finite set of prime divisors of $n_{i}$. Then $B_{S_{i}}$ is a pure subgroup of $B_{\left(S_{i} \backslash P_{i}\right)} \oplus B_{\left(S_{i} \cap P_{i}\right)}$ and

$$
\left(B_{S_{i}}\right)_{\left(S_{i} \backslash P_{i}\right)}=B_{\left(S_{i} \backslash P_{i}\right)}=\left(C_{i}\right)_{\left(S_{i} \backslash P_{i}\right)}=A_{\left(S_{i} \backslash P_{i}\right)} \oplus\left(D_{i}\right)_{\left(S_{i} \backslash P_{i}\right)} .
$$

Let $f: A \rightarrow T$ be the given map which we may assume to be onto so that, for each prime $p, T_{p}$ has finite rank and hence is pure-injective being (divisible) $\oplus$ (finite). Note that $A_{S_{i}}$ is pure in $A_{\left(S_{i} \backslash P_{i}\right)} \oplus A_{\left(S_{i} \cap P_{i}\right)}$. The map $f: A \rightarrow T$ induces $f_{i}$ : $A_{S_{i}} \rightarrow T_{S_{i}}, f_{i}^{\prime}: A_{\left(S_{i} \backslash P_{i}\right)} \rightarrow T_{\left(S_{i} \backslash P_{i}\right)}$, and $f_{i}^{\prime \prime}: A_{\left(S_{i} \cap P_{i}\right)} \rightarrow T_{\left(S_{i} \cap P_{i}\right)}$ for each $i$, such that $\left(f_{i}^{\prime} \oplus f_{i}^{\prime \prime}\right) \mid A_{S_{i}}=f_{i}$. Since $T_{S_{i} \cap P_{i}}=\bigoplus_{p \in S \mathrm{E}_{i} \cap P_{i}} T_{p}$ is pure injective, $f_{i}^{\prime \prime}$ extends to $g_{i}^{\prime \prime}: B_{\left(S_{i} \cap P_{i}\right)} \rightarrow T_{\left(S_{i} \cap P_{i}\right)}$ and, since $\left.A_{\left(S_{i} \backslash P_{i}\right)}\right)$ is a direct summand of $B_{\left(S_{i} \backslash P_{i}\right)}, f_{i}^{\prime}$ extends to $g_{i}^{\prime}: B_{\left(S_{i} \backslash P_{i}\right)} \rightarrow T_{\left(S_{i} \backslash P_{i}\right)}$. Thus $\left(g_{i}^{\prime} \oplus g_{i}^{\prime \prime}\right): B_{\left(S_{i} \backslash P_{i}\right)} \oplus B_{\left(S_{i} \cap P_{i}\right)} \rightarrow T_{S_{i}}$ is an extension of $f_{i}$. Let $g_{i}=\left(g_{i}^{\prime} \oplus g_{i}^{\prime \prime}\right) \mid B_{S_{i}}$. Now $B$ is a pure subgroup of $\oplus_{i=1}^{n} B_{S_{i}}$ and the map $g$ obtained by restricting $g_{1} \oplus \cdots \oplus g_{n}$ to $B$ is the required extension of $f$.

Definition. A group $G$ is said to have the torsion extension property (for short, T.E.P.) if for any pure subgroup $H$ of $G$, every homomorphism $f: H \rightarrow T$, where $T$ is any torsion group, extends to a homomorphism $g: G \rightarrow T$. Equivalently, every pure exact sequence $0 \rightarrow H \rightarrow G \rightarrow K \rightarrow 0$ induces, for any torsion group $T$, the exact sequence $0 \rightarrow \operatorname{Hom}(K, T) \rightarrow \operatorname{Hom}(G, T) \rightarrow \operatorname{Hom}(H, T) \rightarrow 0$.

A mixed abelian group $G$ with the T.E.P. clearly splits with its torsion part being a direct sum of (divisible $\oplus$ bounded) p-groups. The next proposition states an equivalent formulation for torsion-free group with the T.E.P.

Proposition 3. A torsion-free group $G$ has the T.E.P. if and only if for every subgroup $H$ of $G, H^{*} / H$ is a direct summand of $G / H$, where $H^{*}$ is the purfication of $H$ in $G$.

Proof. Suppose $G$ has the T.E.P. Now $H^{*} / H$ is torsion and the natural map $H^{*} \rightarrow H^{*} / H$ extends to a homomorphism $g: G \rightarrow H^{*} / H$. If $K$ is the kernel of $g$, then it is easy to verify that $G / H=H^{*} / H \oplus K / H$. 
Conversely, suppose $A$ is a pure subgroup of $G$. Let $f: A \rightarrow T$ be a homomorphism, where $T$ is any torsion group. Replacing $T$ by $f(A)$, assume $f$ is onto. Now $A$ is the pure subgroup generated by $S=\operatorname{ker} f$. Hence $G / S=A / S \oplus K / S=T \oplus$ $K / S$. If $h: G \rightarrow G / S$ is the natural map and $g: G / S \rightarrow T$ is the coordinate projection, then $g h: G \rightarrow T$ extends $f$.

One may ask if the converse of Theorem 2 is true; that is, whether a finite rank torsion-free group with the T.E.P. must be Butler. The answer is in the negative as the following example (of Butler) shows.

ExAMPLE. Let $\left\{p_{1}, p_{2}, \ldots\right\}$ be an infinite set of primes such that for each $n$, $p_{n}>n^{2}+n$. Let $x_{1}, x_{2}$ be two independent elements of $\mathbf{Q} \oplus \mathbf{Q}$ and let $A$ be the subgroup generated by $\left\{x_{1}, x_{2},\left(x_{1}+n x_{2}\right) / p_{n}: n=1,2, \ldots\right\}$. Then $A$ is a rank two homogeneous group of type $(0,0, \ldots, 0, \ldots)$ and is indecomposable. Since, by [10], a homogeneous Butler group must be completely decomposable, $A$ is not a Butler group. However, $A$ has the T.E.P. since every proper pure subgroup of $A$ is cyclic whose torsion homomorphic images, being finite, are pure injective.

More generally, observe that any torsion-free indecomposable group of finite rank $>1$ whose proper pure subgroups are free will have the T.E.P. but will not be a Butler group. For groups of this type see, e.g., Fuchs [13, p. 125].

It is interesting to note that Theorem 2 is false if $B$ has infinite rank. In other words, an infinite rank Butler group need not have the T.E.P. Indeed, an infinite rank free abelian group will never have the T.E.P. Since there are several nonsplitting mixed groups, the proof of the last statement drops out from the following easily derived reformulation of Proposition 3: A torsion-free group G has the T.E.P. exactly when every epimorphic image of $G$ which is a mixed group splits.

The next section explores an important connection between the T.E.P. and countable Butler groups.

4. Countable rank Butler groups As indicated by Bican and Salce [8], a Butler group $G$ of infinite countable rank is the union of an ascending chain $0=G_{0} \subseteq G_{1}$ $\subseteq \cdots \subseteq G_{n} \subseteq \cdots$ where each $G_{n}$ is a finite rank Butler group and is pure in $G$. Thus availability of sufficiently many finite rank Butler subgroups seems to be essential for the whole group to be a Butler group. This idea is crystalized by the concept of a decent subgroup introduced by Albrecht and Hill [1].

Definition [1]. A subgroup $H$ of a torsion-free group $G$ is said to be decent in $G$ if for any finite subset $S$ of $G$ there is a finite rank Butler subgroup $B$ of $G$ such that $H+B$ is pure in $G$ and contains $S$.

Observe that if $H$ is decent in $G$ so is its purification $H^{*}$. Consequently we may as well assume that our decent subgroups are all pure. This does not lead to any loss in generality in our later arguments. Also notice that in the above definition of the decent subgroup $H$ we may, due to the torsion-freeness of $G$, replace $S$ by a finite rank subgroup $K$ of $G$.

We first explore some of the basic properties of decent subgroups:

LEMMA 4. (i) If $A$ is a decent subgroup of $G$ and $A \subseteq S \subseteq G$, with $S$ pure in $G$, then $A$ is decent in $S$.

(ii) Decency is transitive. 
Proof. (i) Let $X$ be a finite subset of $S$. Now there is a finite rank Butler subgroup $B$ of $G$ such that $A+B$ is pure in $G$ and $X \subseteq A+B$. Then $X \subseteq S \cap(A$ $+B)=A+(S \cap B)$ and $S \cap B$, being pure in $B$, is a Butler group and $A+(S \cap$ $B)=S \cap(A+B)$ is pure in $S$. Hence $A$ is decent in $S$.

(ii) Let $A \subseteq H \subseteq G, A$ a decent subgroup of $H$ and $H$ a decent subgroup of $G$. Let $X$ be a finite subset of $G$. Then $X \subseteq H+B$, where $B$ is a finite rank Butler subgroup of $G$ and $H+B$ is pure in $G$. Also there exists $B_{1} \subset H, B_{1}$ a finite rank Butler group such that $A+B_{1}$ is pure in $H$ and $(H \cap B) \cup Y \subseteq A+B_{1}$ where $Y$ is the finite set of $H$-components of elements of $X \subset H+B$. We claim that $A+B_{1}+B$ is pure in $G$. Let $x=a+b_{1}+b \in\left(A+B_{1}+B\right) \cap n G$. By the purity of $H+B, x=n h+n b^{*}$, where $h \in H, b^{*} \in B$. Then

$$
n h-a-b_{1}=n b^{*}-b \in H \cap B \subseteq A+B_{1} .
$$

It follows that $n h \in A+B_{1}$. By the purity of $A+B_{1}$ in $H, n h=n\left(a^{*}+b_{1}^{*}\right)$ for $a^{*} \in A, b_{1}^{*} \in B$. Then

$$
x=n h+n b^{*}=n\left(a^{*}+b_{1}^{*}+b^{*}\right) \in A+B_{1}+B .
$$

Hence $A+B_{1}+B$ is a pure subgroup of $G$ containing $X$ and $B_{1}+B$ is a Butler group of finite rank. Thus $A$ is decent in $G$.

It is clear that a finite rank torsion-free group $G$ is Butler exactly when every pure subgroup is decent in $G$. Note that this does not hold for infinite rank Butler groups. For instance if $A$ is a finite rank group which is not free and if we write $A=F / K$, where $F$ is a free group of countable rank, then $K$ is pure but cannot be decent in $F$.

Proposition 5. A torsion-free group $G$ is a $B_{2}$-group if and only if $G$ has a smooth $\kappa$-filtration, $G=\cup_{\alpha<\kappa} G_{\alpha}$ where $\left|G_{\alpha}\right|<\kappa$ for all $\alpha<\kappa$ and $G_{\alpha}$ are pure decent subgroups of $G$ such that for each $\alpha, G_{\alpha+1} / G_{\alpha}$ is countable.

Proof. Suppose $G$ is a $B_{2}$-group so that $G$ has a smooth filtration $G=\cup_{\alpha<\kappa} G_{\alpha}$ where, for each $\alpha, G_{\alpha}$ is pure in $G, G_{\alpha+1}=G_{\alpha}+B_{\alpha}, B_{\alpha}$ a finite rank Butler group. Clearly $G_{\alpha}$ is decent in $G_{\alpha+1}$. Lemma 4(ii) above and a simple transfinite induction imply that $G_{\alpha}$ is decent in $G$ for all $\alpha$. Obviously $G_{\alpha+1} / G_{\alpha}$ is countable.

Conversely, suppose $G=\cup_{\alpha<\kappa} G_{\alpha}$ is a smooth $\kappa$-filtration where, for each $\alpha, G_{\alpha}$ is pure and decent in $G$ and $G_{\alpha+1} / G_{\alpha}$ is countable. Since $G_{\alpha+1}=G_{\alpha}+X$, where $X$ is a countable subgroup and since $G_{\alpha}$ is decent (in $G$ and hence by Lemma 4(i)) in $G_{\alpha+1}$, we get a sequence $B_{1}, B_{2}, \ldots$ of Butler groups of finite rank such that $G_{\alpha}+B_{n} \subseteq G_{\alpha}+B_{n+1}$ is pure for all $n=1,2, \ldots$ and $G_{\alpha+\omega}=\bigcup_{n<\omega}\left(G_{\alpha}+B_{n}\right)$. Then the filtration $G=\cup_{\alpha<\kappa} G_{\alpha}$ can be refined to a smooth filtration $G=\cup_{\gamma<\kappa} G_{\gamma}$ where, for each $\gamma, G_{\gamma}$ is pure in $G$ and $G_{\gamma+1}=G_{\gamma}+B_{\gamma}$, with $B_{\gamma}$ a finite rank Butler group. Hence $G$ is a $B_{2}$-group.

Since for a group $G$ of cardinality $\aleph_{1}$, a smooth filtration $G=\bigcup_{\alpha<\omega_{1}} G_{\alpha}$ of countable subgroups is an Axiom 3 family (see [1]) we deduce the following special case of an important theorem of Albrecht and Hill [1]. 
Corollary [1]. Suppose $G$ is a torsion-free group of cardinality $\boldsymbol{\aleph}_{1}$. Then $G$ is a $B_{2}$-group if and only if $G$ satisfies Axiom 3 with respect to decent subgroups.

We wish to explore the interaction between the T.E.P. and the decency of a subgroup. For convenience of expression, we introduce the following

Definition. A group $G$ satisfies the T.E.P. over a pure subgroup $H$ if every homomorphism $f: H \rightarrow T$, where $T$ is any torsion group, extends to a $g: G \rightarrow T$.

The next proposition says that, in a $p$-local Butler group, the T.E.P. over a pure subgroup $H$ implies the decency of $H$.

Proposition 6. Let $G$ be a p-local completely decomposable group and $A$ a pure subgroup of $G$. If $G$ has the T.E.P. over $A$, then $A$ is decent in $G$.

Proof. If $A$ has finite rank, then $A$ is a direct summand of $G$ and hence is decent. Hence we may assume that $A$ has infinite rank. Suppose, by way of contradiction, $A$ is not decent in $G$. Then there exists a pure subgroup $L$ of $G$ containing $A$ such that $A$ is not decent in $L$ and $L / A$ has finite rank. We may choose $L$ with the rank of $L / A$ minimal. Then there exists a pure subgroup $H$ with $A \subseteq H \subseteq L$ such that $L / H$ has rank one and $A$ is decent in $H$. Now $H=A+C^{\prime}$ where $C^{\prime}$ is a finite rank pure subgroup of $G$. Moreover, $C^{\prime}$ is completely decomposable so that $A \cap C^{\prime}$ is a summand of $C^{\prime}$. Hence $H=A \oplus C$, where $\left(A \cap C^{\prime}\right) \oplus C=C^{\prime}$. We claim that $H$ is a direct summand of $L$. Without loss of generality, we may assume that $L$ is reduced, so that $L$ and $A$ are free $\mathbf{Z}_{p}$-modules. Note that $L / H=\mathbf{Z}_{p}$ or $\mathbf{Q}$. By way of contradiction assume that $H$ is not a summand of $L$. Then $L / H=\mathbf{Q}$ so that $L \subseteq \hat{H}=\hat{A} \oplus \hat{C}$ where ^ denotes the $p$-adic completion. Since $\hat{L}$ has infinite rank as a $\mathbf{Z}_{p}$-module, there is no finite rank summand $D$ of $A$ such that $L \subseteq \hat{D} \oplus \hat{C}$. Hence we can find a $y \in L$ such that

$$
y=\sum_{i=1}^{\infty} p^{n_{i}} k_{i} a_{i}+x,
$$

where $x \in C$, the $k_{i}$ 's are integers not divisible by $p$, and the set $\left\{a_{i}: i=1,2, \ldots\right\}$ is contained in a fixed basis of the free $\mathbf{Z}_{p}$-module $A$. Consider a $p$-group $T=$ $\oplus_{k<\omega} \mathbf{Z}\left(p^{k}\right)$. For each $i$, choose a basis element $e_{i}$ in $T$ such that $o\left(e_{i}\right)>n_{i}+i$. If $f: A \rightarrow T$ is a homomorphism given by $f\left(a_{i}\right)=e_{i}$ and $f$ vanishes on the other elements of the fixed basis of $A$, then, by hypothesis, $f$ extends to $g: L \rightarrow T$. By continuity $g$ extends to $g^{\prime}: \hat{L} \rightarrow \hat{T}$. Since $C$ has finite rank, $g(C)$ is bounded, say $p^{m}(g(C))=0$ for some positive integer $m$. Then $g\left(p^{m} y\right)=g^{\prime}\left(p^{m} y\right)=$ $\sum_{i=1}^{\infty} p^{n_{i}+m} k_{i} e_{i} \notin T$ since, for all $i \geqslant m, p^{n_{i}+m} k_{i} e_{i} \neq 0$. This is a contradiction. Hence $H$ and therefore $A$ must be a summand of $L$. But this implies that $A$ is decent in $L$ contrary to our assumption. Hence $A$ must be decent in $G$.

Corollary 1. Suppose $G$ is p-local completely decomposable and $A$ is pure in $G$. If $G$ has the T.E.P. over $A$ and further $G / A$ has finite rank, then $A$ is a direct summand of $G$. 
Proof. By Proposition 6, $A$ is decent in $G$. Since $G / A$ has finite rank, $G=A+C$, where $C$ can be taken to be a finite rank completely decomposable pure subgroup of $G$. Now $A \cap C$, being pure in $C$, is a summand: $C=(A \cap C) \oplus C^{\prime}$. Then $G=A \oplus$ $C^{\prime}$.

Proposition 6 also yields the following corollary which is used in the proof of the next theorem.

Corollary 2. Let $G$ be a Butler group and $A$ be a pure subgroup of $G$ with $G / A$ having finite rank. If $G$ has the T.E.P. over $A$, then for each prime $p$ there exists $a$ finite rank subgroup $C$ of $G$ such that $A+C$ is p-pure in $G$ and $G /(A+C)$ is torsion.

Proof. Since $G$ is Butler, the localization $G_{p}$ is completely decomposable. Also $G_{p}$ has the T.E.P. over $A_{p}$. By Corollary 1 above, $G_{p}=A_{p} \oplus \oplus_{i=1}^{n}\left(\mathbf{Z}_{p} \otimes\left\langle x_{i}\right\rangle_{*}\right)$, where $x_{i} \in G$. Let $C=\left\langle x_{i}: i=1,2, \ldots, n\right\rangle_{*}$. Now tensoring the exact sequence $0 \rightarrow A+$ $C \rightarrow G \rightarrow G /(A+C) \rightarrow 0$ by $\mathbf{Z}_{p}$ we get the exact sequence

$$
0 \rightarrow \mathbf{Z}_{p} \otimes(A+C) \rightarrow \mathbf{Z}_{p} \otimes G \rightarrow \mathbf{Z}_{p} \otimes(G /(A+C)) \rightarrow 0 .
$$

Since $\mathbf{Z}_{p} \otimes(A+C)=\mathbf{Z}_{p} \otimes G$, we have $\mathbf{Z}_{p} \otimes(G /(A+C))=0$. This implies that $G /(A+C)$ is a torsion group with its $p$-primary part zero. That is, $A+C$ is a full $p$-pure subgroup of $G$.

We are now ready to prove the main theorem of this section. This result is indeed the kingpin of the entire paper. This theorem also has turned out to be crucial in our subsequent papers.

THEOREM 7. Let $B$ be a Butler group of rank $\boldsymbol{\aleph}_{0}$ and $A$ a pure subgroup. Then $A$ is decent in $B$ if and only if $B$ satisfies the T.E.P. over $A$.

Proof. Suppose $A$ is decent in $B$. Since $B$ is countable we can easily construct an increasing chain of pure subgroups $A=A_{0} \subseteq A_{1} \subseteq A_{2} \subseteq \cdots$ such that $B=$ $\cup_{n<\omega} A_{n}$ and, for each $n, A_{n+1} / A_{n}$ has finite rank. Since $A$ is decent in $A_{n}$ by Lemma 4(i), we have $A_{n}=A+C_{n}$ for each $n$, where $C_{n}$ is a finite rank Butler group. It is then easily seen that $A_{n}$ is decent in $A_{n+1}$ and since $A_{n+1} / A_{n}$ has finite rank, we can write $A_{n+1}=A_{n}+B_{n}$, where $B_{n}$ is a Butler group of finite rank. Let $f$ : $A \rightarrow T$ be a homomorphism, where $T$ is any torsion group. Suppose, for some $n \geqslant 0$, we have an extension $f_{n}: A_{n} \rightarrow T$. Now, by Theorem 2 , the restriction $f_{n} \mid\left(A_{n} \cap B_{n}\right): A_{n} \cap B_{n} \rightarrow T$ extends to a homomorphism $h: B_{n} \rightarrow T$. Then $f_{n+1}$ : $A_{n+1} \rightarrow T$ given by $f_{n+1}(a+b)=f_{n}(a)+h(b)$ for all $a \in A_{n}, b \in B_{n}$ is a homomorphism satisfying $f_{n+1} \mid A_{n}=f_{n}$. By an induction argument we can then construct a homomorphism $g: B \rightarrow T$ such that $g \mid A=f$.

Conversely, suppose that $B$ satisfies the T.E.P. over $A$. Suppose, by way of contradiction, that $A$ is not decent in $B$. Then there is a pure subgroup $L$ containing $A$ such that $L / A$ has finite rank and $A$ is not decent in $L$. Since $L$ is also a Butler group [4], we may replace $L$ by $B$ and assume that $B / A$ has finite rank. We shall be frequently using the fact that any finite rank pure subgroup of $B$ is Butler [4].

First observe that if $R$ is a finite rank subgroup with $B /(A+R)$ torsion and if $M_{p} /(A+R)$ is the $p$-component of $B /(A+R)$, then $M_{p}=A+X$ for some finite rank subgroup $X$. This is because, by Corollary 2 to Proposition 6 , there is a finite 
rank subgroup $C$ such that $A+C$ is $p$-pure in $B$ and $B /(A+C)$ is torsion. Since $A+R+C$ is still $p$-pure in $B$ and contains $M_{p}$, we get, by modularity, $M_{p}=A+$ $R+\left(M_{p} \cap C\right)=A+X$, where $X=R+\left(M_{p} \cap C\right)$ has finite rank. Since, by the indecency of $A, B$ is not of the form $B=A+Y$, for any finite rank subgroup $Y$, the last statement implies:

To each finite rank subgroup $R$ with $B /(A+R)$ torsion, there are infinitely many primes $p$ such that $A+R$ is not $p$-pure in $B$.

Since $A$ is a Butler group of countable rank, we may write $A=\cup_{n \geqslant 0} A_{n}$, where for each $n, A_{n} \subseteq A_{n+1}$ and $A_{n}$ is pure and a Butler group of finite rank [8]. Let $B / A=\left\langle b_{i}+A: 1 \leqslant i \leqslant r\right\rangle_{*}, r$ being the rank of $B / A$. Let $K_{0}=\left\langle b_{i}: 1 \leqslant i \leqslant r\right\rangle$ and for $n>0, K_{n}=A_{n}+K_{n-1}^{*}$, where $K_{n-1}^{*}$ is the purification of $K_{n-1}$ in $B$.

We distinguish two cases:

Suppose there is an integer $n_{0}$ such that for all $n \geqslant n_{0}$ there is an $i>1$ with $A_{n+i}+K_{n+i-1}^{*}$ a generalized regular subgroup of its purification in $B$. Passing on to a subsequence of the $A_{n}$ 's, we may state our first case as

Case 1. There is an integer $n_{0}$ such that for each $n \geqslant n_{0}, K_{n+1}=A_{n+1}+K_{n}^{*}$ is a generalized regular subgroup of its purification $K_{n+1}^{*}$.

Since $K_{n+1}^{*}$ is a finite rank Butler group we conclude, by Bican [6], that the set $\Pi_{n}=\left\{p \in \mathbf{P}: K_{n+1}\right.$ is not $p$-pure in $\left.B\right\}$ is finite for all $n$, but that $\bigcup_{n=0}^{\infty} \Pi_{n}$ is infinite.

In view of (I), by induction on $n \geqslant n_{0}$, we could find elements $c_{n} \in A_{n} \backslash A_{n-1}$, $k_{n} \in K_{n-1}^{*}$ and primes $p_{n} \in \Pi_{n-1}$ such that

(i) $p_{n}$ divides $c_{n}+k_{n}$ in $B$ but not in $K_{n}$,

(ii) $p_{n} \notin \bigcup_{i=n_{0}}^{n-2} \Pi_{i}$.

Observe that the above choice of $c_{n}$ 's is made by passing, if necessary, to a subsequence of the $A_{n}$ 's. Note also that, for all $n, h_{p_{n}}^{B}\left(c_{n}\right)=0$.

We shall next show that

(iii) For all $n, h_{p_{n}}\left(c_{n}+A_{n-1}\right)=0$ in $A_{n} / A_{n-1}$.

Suppose $h_{p_{n}}\left(c_{n}+A_{n-1}\right)>0$. Then there is a $y \in A_{n-1}$ such that $h_{p_{n}}^{A}\left(c_{n}+y\right)>0$. Since, by (i), $h_{p_{n}}^{B}\left(c_{n}+k_{n}\right)>0$, we get $h_{p_{n}}^{B}\left(k_{n}-y\right)>0$. Since $K_{n-1}$ is $p_{n}$-pure in $K_{n-1}^{*}$, there is an integer $z$ not divisible by $p_{n}$ such that $z\left(k_{n}-y\right) \in K_{n-1}$. For the same reason $h_{p_{n}}^{K_{n-1}}\left(z\left(k_{n}-y\right)\right)>0$. Then $z\left(c_{n}+k_{n}\right)=z\left(c_{n}+y\right)+z\left(k_{n}-y\right) \in$ $p_{n}\left(A_{n}+K_{n-1}\right)$ so that $h_{p_{n}}^{K_{n}}\left(c_{n}+k_{n}\right)>0$, a contradiction to (i) above. This proves (iii).

We will now define a homomorphism $f: A \rightarrow T$, where $T$ is a torsion group such that, for each prime $p$, its $p$-component $T_{p}=\bigoplus_{i=0}^{\infty} T_{p, i}$, with each $T_{p, i}$ being an unbounded direct sum of cyclic $p$-groups. This map $f$ is constructed by defining, for each $n \geqslant n_{0}$, a homomorphism $f_{n}: A_{n} \rightarrow T$ such that $f_{n+1} \mid A_{n}=f_{n}$ and, moreover, will have the following properties:

(Recall that $K_{n}=A_{n}+\left\langle\left\{A_{n-1}, b_{1}, b_{2}, \ldots, b_{r}\right\}\right\rangle_{*}$. For convenience of notation, we will write $f$ in place of $f_{n}$ and this should not. hopefully, cause any confusion. Also for an element $x$ in a torsion group $T$ and any prime $p, x_{p}$ denotes the $p$-component of $x$.) 


$$
f\left(A_{n-1}\right)_{p_{n-1}} \subseteq \bigoplus_{i=0}^{\bar{k}_{n-1}} T_{p_{n-1}, i}
$$

$f\left(c_{n}\right)_{p_{n}}$ is an element of a fixed basis of $T_{p_{n}, r_{n}}$ with $r_{n}>\tilde{k}_{n-1}$ and $o\left(f\left(c_{n}\right)\right)>h_{p_{n}}^{B}\left(b_{i}\right)$ whenever $h_{p_{n}}^{B}\left(b_{i}\right)$ is finite, $1 \leqslant i \leqslant r$,

For the element $c_{n_{0}} \in A_{n_{0}}$, we can define $f:\left\langle c_{n_{0}}\right\rangle_{*} \rightarrow T$ satisfying $(\alpha)$ and $(\beta)$ since $h_{p_{n_{0}}}^{A}\left(c_{n_{0}}\right)=0$. By Theorem 2, $f$ extends to a map $A_{n_{0}} \rightarrow T$ which we call $f$ again.

Suppose $f$ is already defined on $A_{n-1}$ satisfying $(\alpha),(\beta)$, and $(\gamma)$. By Theorem 2, this map $f$ lifts to $f^{\prime}: A_{n} \rightarrow T$.

We define $g: A_{n} / A_{n-1} \rightarrow T$ such that $g\left(c_{n}+A_{n-1}\right)$ satisfies $(\beta)$. This can be done because of (iii). Let $g^{\prime}: A_{n} \rightarrow T$ denote the map obtained by composing the natural map $A_{n} \rightarrow A_{n} / A_{n-1}$ with the map $g$. Then define $f \mid A_{n}=f^{\prime}+g^{\prime}$ which clearly satisfies $(\alpha),(\beta)$, and $(\gamma)$. By hypothesis the map $f$ extends to $f^{*}: B \rightarrow T$.

Now $k_{n} \in K_{n-1}^{*} \subseteq \bar{A}_{n-1}+\left\{\overline{b_{i}: 1 \leqslant i \leqslant r}\right\}$, where $\bar{G}$ denotes the divisible hull of $G$. Since $K_{n-1}$ is $p_{n}$-pure in $K_{n-1}^{*}$, there is a nonzero integer $q$ not divisible by $p_{n}$ such that $q k_{n} \in K_{n-1}$, say, $q k_{n}=a_{n-1}+\sum_{i=1}^{r}\left(r_{i} / s_{i}\right) b_{i}$ where $a_{n-1} \in A_{n-1}, r_{i}, s_{i}$ are integers, and $\left|s_{i}\right|_{p_{n}} \leqslant h_{p_{n}}^{B}\left(b_{i}\right)$. (Here $\left|s_{i}\right|_{p_{n}}$ denotes the exponent of the highest power of $p_{n}$ that divides $s_{i}$.) Let $E=\left\{j: 1 \leqslant j \leqslant r\right.$ and $b_{j}$ has finite $p_{n}$-height $\}$. Let $e=\max \left\{\left|s_{i}\right|_{p_{n}}: i \in E\right\}$. Then $p_{n}^{e} q k_{n}=p_{n}^{e} a_{n-1}+\sum_{i=1}^{r} q_{n, i} b_{i}$ where $p_{n}$ does not divide the denominator of $q_{n, i}=p_{n}^{e} r_{i} / s_{i}$. Note that if $i \notin E, b_{i}$ has infinite $p_{n}$-height and since $T$ has no nonzero element of infinite height $f^{*}\left(b_{i}\right)_{p_{n}}=0$ for all $i \notin E$. Then we obtain

$$
\begin{aligned}
f^{*}\left(p_{n}^{e} q\left(c_{n}+k_{n}\right)\right)_{p_{n}} & =f^{*}\left(p_{n}^{e} q c_{n}\right)_{p_{n}}+f^{*}\left(p^{e} q k_{n}\right)_{p_{n}} \\
& =f^{*}\left(p_{n}^{e} q c_{n}\right)_{p_{n}}+f\left(p_{n}^{e} a_{n-1}\right)_{p_{n}}+\sum_{i \in E} q_{n i} f^{*}\left(b_{i}\right)_{p_{n}} .
\end{aligned}
$$

Now

$$
h_{p_{n}}^{T_{p_{n}}}\left(f^{*}\left(p_{n}^{e} q\left(c_{n}+k_{n}\right)\right)_{p_{n}}\right) \geqslant h_{p_{n}}^{K_{*}^{*}}\left(p_{n}^{e} q\left(c_{n}+k_{n}\right)\right)>e
$$

by (i) and $h_{p_{n}}^{T}\left(f\left(p_{n}^{e} q c_{n}\right)+f\left(p_{n}^{e} a_{n-1}\right)\right)=e$ since, by $(\alpha)$ and $(\beta), c_{n}$ and $A_{n-1}$ are mapped into complementary summands. Thus for some $i, q_{n i} f^{*}\left(b_{i}\right)_{p_{n}} \neq 0$. Since $p_{n}$ does not divide the denominator of $q_{n i}, f^{*}\left(b_{i}\right)_{p_{n}} \neq 0$. Since this holds for infinitely many $p_{n}$ 's and since there are only a finite number of $b_{i}$ 's, we conclude that there is an $i_{0}$ such that $f^{*}\left(b_{i_{0}}\right)_{p_{n}} \neq 0$ for infinitely many $p_{n}$ 's. But this is impossible since $f^{*}\left(b_{i_{0}}\right) \in T$, a torsion group. This contradiction shows that $A$ has got to be decent in $B$.

Case 2. Suppose, for infinitely many $n, K_{n}=A_{n}+K_{n-1}^{*}$ is not generalized regular in $K_{n}^{*}$.

Rewriting the filtration $A=\cup A_{n}$ we may assume without loss of generality that, for each $n, K_{n}$ is not generalized regular in $K_{n}^{*}$.

Then there exists $c_{n} \in A_{n} \backslash A_{n-1}, y_{n} \in K_{n-1}^{*}$, and an infinite set of primes $P_{n}$ such that, for all $p \in P_{n}, h_{p}^{K_{n}}\left(c_{n}+y_{n}\right)<h_{p}^{B}\left(c_{n}+y_{n}\right)=m_{p}$, say. We claim that

(*) the set $\bar{P}_{n}=\left\{p \in P_{n}: h_{p}\left(c_{n}+A_{n-1}\right) \geqslant m_{p}\right.$ in $\left.A_{n} / A_{n-1}\right\}$ is finite. 
Suppose, by way of contradiction, the set $\bar{P}_{n}$ is infinite. Since $A_{n}$ is finite rank Butler, we get from the proof of Theorem 2 a partition $S_{1} \cup \cdots \cup S_{k}$ of the set $\mathbf{P}$ of all primes and finite subsets $L_{j} \subseteq S_{j}$ for $j=1, \ldots, k$ such that the localization $\left(A_{n-1}\right)_{S_{j} \backslash L_{j}}$ is a direct summand of $\left(A_{n}\right)_{S_{j} \backslash L_{j}}$. Now for some index, say $i, \bar{P}_{n} \cap S_{i}$ is infinite. Write $\left(A_{n}\right)_{S_{i} \backslash L_{i}}=\left(A_{n-1}\right)_{S_{i} \backslash L_{i}} \oplus D_{i}$. Let $c_{n}=c_{n} \otimes 1 \in\left(A_{n}\right)_{S_{i} \backslash L_{i}}$. Then $c_{n}=a_{(n-1), i}+x_{n, i}$ where $x_{n, i} \in D_{i}, a_{(n-1), i} \in\left(A_{n-1}\right)_{S_{i} \backslash L_{i}}$ and for all $p \in \bar{P}_{n} \cap$ $\left(S_{i} \backslash L_{i}\right), h_{p}^{D_{i}}\left(x_{n, i}\right) \geqslant m_{p}$. There exists an integer $t_{i}$ relatively prime to all primes in $S_{i} \backslash L_{i}$ such that $t_{i} x_{n, i} \in A_{n}$ and $t_{i} a_{(n-1), i} \in A_{n-1}$. Then

$$
\begin{aligned}
t_{i}\left(y_{n}+a_{(n-1), i}\right) & =t_{i}\left(c_{n}+y_{n}\right)-t_{i}\left(c_{n}-a_{(n-1), i}\right) \\
& =t_{i}\left(c_{n}+y_{n}\right)-t_{i} x_{n, i} \in p^{m_{p}} B \cap K_{n-1}^{*}=p^{m_{p}} K_{n-1}^{*} .
\end{aligned}
$$

Since $t_{i} x_{n, i} \in p^{m_{p}} A_{n}$ we also get that $t_{i}\left(c_{n}+y_{n}\right) \in p^{m_{p}} A_{n}+p^{m_{p}} K_{n-1}^{*}=p^{m_{p}} K_{n}$. Since $p$ does not divide $t_{i}, c_{n}+y_{n} \in p^{m_{p}} K_{n}$ for all $p \in \bar{P}_{n} \backslash L_{i}$. This contradicts the choice of $P_{n}$. Hence $\bar{P}_{n} \cap\left(S_{i} \backslash L_{i}\right)$ is empty, thus proving that $\bar{P}_{n}$ must be finite.

For the sake of convenience rename $P_{n}=$ primes $p: m_{p}>0$ and $h_{p}\left(c_{n}+A_{n-1}\right)$ $<m_{p}$ in $\left.A_{n} / A_{n-1}\right\}$. In view of $(*), P_{n}$ is still infinite.

Let $\bar{B}$ be the divisible hull of $B$. Then $\bar{B}=\bar{A} \oplus \sum_{i=1}^{r} \mathbf{Q} b_{i}$. The element $y_{n} \in K_{n-1}^{*}$ has a representation in $\bar{B}$ as $y_{n}=s_{n} a_{n}+\sum_{i=1}^{s} s_{n_{i}} b_{i}$ with $s_{n}, s_{n_{i}} \in \mathbf{Q}$ and $a_{n} \in A_{n-1}$. Let $W_{n}$ be the finite set of primes that show up in the numerators or denominators of $s_{n}$ and $s_{n i}$. Then $\tilde{P}_{n}=P_{n} \backslash W_{n}$ is an infinite set.

By induction on $n$ we wish to choose $p_{n} \in \tilde{P}_{n}$ and define a homomorphism $f_{n}$ : $A_{n} \rightarrow T, T$ a torsion group such that

(i) $f_{n} \mid A_{n-1}=f_{n-1}$,

(ii) $f_{n}\left(a_{n}\right)\left(=f_{n-1}\left(a_{n}\right)\right)$ has $p_{n}$-component zero,

(iii) $f_{n}\left(c_{n}\right)$ has $p_{n}$-height $<m_{p_{n}}$.

Here $T$ is chosen as before, namely, for each prime $p$, the $p$-component $T_{p}=$ $\oplus_{i<\omega} T_{p, i}$ where each $T_{p, i}$ is an unbounded direct sum of cyclic $p$-groups.

Suppose $f_{n-1}$ has been defined already satisfying (i), (ii), and (iii). Since $f_{n-1}\left(a_{n}\right)$ $\in T$, we can find $p_{n} \in P_{n}$ with $f_{n-1}\left(a_{n}\right)$ having $p_{n}$-component zero. By Theorem 2, $f_{n-1}$ extends to $f_{n-1}^{\prime}: A_{n} \rightarrow T$. If $f_{n-1}^{\prime}$ satisfies (iii), we are done. Suppose on the other hand $f_{n-1}^{\prime}\left(c_{n}\right)$ has $p_{n}$-height $\geqslant m_{p_{n}}$. Since $h_{p_{n}}\left(c_{n}+A_{n-1}\right)<m_{p_{n}}$, we then can define using Theorem 2 a map $g: A_{n} / A_{n-1} \rightarrow T$ such that $h_{p_{n}}\left(g\left(c_{n}\right)\right)<m_{p_{n}}$. If $g^{\prime}$ : $A_{n} \rightarrow T$ is obtained by composing the natural map $A_{n} \rightarrow A_{n} / A_{n-1}$ with $g$, then $f_{n}=f_{n-1}^{\prime}+g^{\prime}: A_{n} \rightarrow T$ satisfies properties (i), (ii), and (iii). Define $f=\bigcup_{n=1}^{\infty} f_{n}$. Let $\bar{f}: \bar{B} \rightarrow \bar{T}$ be the extension of $f$ to $\bar{B}$.

We now have, for each $p_{n} \in P_{n} \backslash W_{n}$,

$$
\begin{aligned}
m_{p_{n}} & \leqslant h_{p_{n}}\left(\bar{f}\left(c_{n}+y_{n}\right)_{p_{n}}\right)=h_{p_{n}}\left(\bar{f}\left(c_{n}+s_{n} a_{n}+\sum_{i=1}^{r} s_{n i} b_{i}\right)\right) \\
& =h_{p_{n}}\left[\left(\bar{f}\left(c_{n}\right)_{p_{n}}\right)+s_{n} \bar{f}\left(a_{n}\right)+\sum_{i=1}^{r} s_{n_{i}} \bar{f}\left(b_{i}\right)\right] .
\end{aligned}
$$


Because of (ii), $\left(\bar{f}\left(a_{n}\right)\right)_{p_{n}}=0$ and by (iii), $h_{p_{n}}\left(\bar{f}\left(c_{n}\right)\right)<m_{p_{n}}$. Hence there is at least one $i=i(n)$ with $\bar{f}\left(b_{i}\right)_{p_{n}} \neq 0$. Since there are infinitely many $n$ 's and only finitely many $b_{i}$, for some $i=i_{0}, \bar{f}\left(b_{i_{0}}\right)_{p_{n}} \neq 0$ for infinitely many $n$. This is impossible since $\bar{f}\left(b_{i_{0}}\right) \in \bar{T}$, a torsion group. This contradiction completes the proof.

As a corollary to Theorem 7 we obtain an alternative proof of the theorem of Bican [6]:

COROLlaRy [6]. A finite rank torsion-free group $G$ is Butler in the classical sense, that is, $G=R_{1}+\cdots+R_{n}, R_{i}$ rank one groups if and only if $\operatorname{Bext}(G, T)=0$ for all torsion groups.

Proof. Consider a balanced exact sequence $0 \rightarrow A \rightarrow B \rightarrow G \rightarrow 0$ where $B$ is a countable rank completely decomposable group, say, $B=\oplus_{x \in G}\langle x\rangle_{*}$. For any torsion group $T$, we get the induced exact sequence $\operatorname{Hom}(B, T) \rightarrow \operatorname{Hom}(A, T) \rightarrow$ $\operatorname{Bext}(G, T) \rightarrow 0$. Then $\operatorname{Bext}(G, T)=0$ if and only if $B$ satisfies the T.E.P. over $A$. By Theorem 7, this happens exactly when $A$ is decent in $B$. Since $A$ is balanced in $B$, the last statement is equivalent to saying that $G=R_{1}+\cdots+R_{n}$ where each $R_{i}$ is a rank one group.

5. Butler groups of uncountable rank. In this section we consider torsion-free groups of cardinality $\aleph_{1}$.

D. Arnold [4] and Bican and Salce [8] raised the question whether the classes of groups $\mathscr{B}_{1}$ and $\mathscr{B}_{2}$ referred to in the introduction are identical. This question is also mentioned in Albrecht and Hill [1]. Employing Gödel's axiom of constructibility $(V=L)$, we are able to show that a Butler group of rank $\boldsymbol{\aleph}_{1}$ in which every finite rank pure subgroup is Butler is a $B_{2}$-group. Actually we prove a stronger statement, namely, that under $V=L$, countable torsion groups are enough to test whether a torsion-free group is Butler. On the other hand, under Martin's axiom and the negation of the continuum hypothesis, we give an example of a group $B$ such that $\operatorname{Bext}(B, T)=0$ for all countable torsion groups $T$, but $B$ is neither in $\mathscr{B}_{2}$ nor in $\mathscr{B}_{3}$. Thus the problem whether $\mathscr{B}_{2}$ coincides with $\mathscr{B}_{1} \cap \mathscr{B}_{3}$ is undecidable in standard ZFC if one restricts the class of torsion groups $T$ in the definition of $\mathscr{B}_{1}$ to countable groups.

THEOREM $8(V=L)$. Let $B$ be a Butler group of rank $\aleph_{1}$ such that every finite rank pure subgroup of $B$ is again Butler. Then $B$ is a $B_{2}$-group.

Proof. We wish to prove that $B$ has a smooth filtration $B=\bigcup_{\alpha<\omega_{1}} B_{\alpha}$ where each $B_{\alpha}$ is countable, pure and decent in $B$. We start with any pure $\omega_{1}$-filtration $B=\bigcup_{\alpha<\omega_{1}} B_{\alpha}$ of $B$. By way of contradiction, suppose $E=\left\{\alpha<\omega_{1}: B_{\alpha}\right.$ not decent in $B$ \} is a stationary set. Since $B_{\alpha}$ is not decent in $B$, there exists a pure subgroup $H$ such that $B_{\alpha}$ is not decent in $H$ and $H / B_{\alpha}$ has finite rank. Changing the filtration we may assume that $H=B_{\alpha+1}$. Let $T$ be a countable torsion group.

We wish to construct a mixed group $G$ by means of a filtration $G=\cup_{\alpha<\omega_{1}} G_{\alpha}$ with embeddings $j_{\alpha}: G_{\alpha} \rightarrow G_{\alpha+1}$ such that $0 \rightarrow T \rightarrow G \rightarrow B \rightarrow 0$ is balanced exact and $G_{\alpha}=T \oplus B_{\alpha}$ for each $\alpha$. Observe that for $G_{\lambda}=\bigcup_{\alpha<\lambda} T+B_{\alpha}$ we have that $T$ is 
balanced in $G_{\lambda}$ and since $B_{\lambda}$ is Butler, $G_{\lambda}=T \oplus B_{\lambda}$. Let $\left\{\phi_{\alpha}: B_{\alpha} \rightarrow T, \alpha \in E\right\}$ be the set of Jensen functions provided by $\diamond_{E^{\left(\omega_{1}\right)}}$ (see [16]). Suppose $G_{\alpha}$ has already been constructed. We distinguish two cases:

Case 1. $\alpha \in E$ and $\phi_{\alpha}: B_{\alpha} \rightarrow T$ is a homomorphism.

Theorem 7 guarantees the existence of an $f_{\alpha} \in \operatorname{Hom}\left(B_{\alpha}, T\right)$ such that there is no $g_{\alpha} \in \operatorname{Hom}\left(B_{\alpha+1}, T\right)$ for which $g_{\alpha} \mid B_{\alpha}=f_{\alpha}$. Now define $j_{\alpha}$ by

$$
j_{\alpha}((t, b))=\left(t+\left(f_{\alpha}-\phi_{\alpha}\right)(b), b\right) \text { for all } t \in T, b \in B_{\alpha} .
$$

Then $j_{\alpha}: T \oplus B_{\alpha} \rightarrow T \oplus B_{\alpha+1}$ is an embedding.

Case 2. If Case 1 does not apply, then define $j_{\alpha}=1_{T}+i$ where $i$ is the natural embedding $i: B_{\alpha} \rightarrow B_{\alpha+1}$ and $1_{T}$ is the identity map on $T$.

Define $G_{\alpha+1}=T \oplus B_{\alpha+1}$. Then $G=\bigcup_{\alpha<\omega_{1}} G_{\alpha}$ is the required filtration with the embeddings $j_{\alpha}$. Clearly $T$ is balanced in $G$ and $G / T \cong B$. Since $\operatorname{Bext}(B, T)=0, G$ splits, say, $G=T \oplus B^{\prime}, B^{\prime} \cong B$ and $B^{\prime}=\{(\phi(b), b): b \in B\}$ for some $\phi \in$ $\operatorname{Hom}(B, T)$. Since $\diamond_{E^{\left(\omega_{1}\right)}}$ holds, the set

$$
E^{\prime}=\left\{\alpha \in E: \phi_{\alpha}=\phi \mid B\right\}
$$

is stationary in $\omega_{1}$. Let $\alpha \in E^{\prime}$. Then we obtain $G_{\alpha}=T \oplus\left(B^{\prime} \cap G_{\alpha}\right)$ is contained in $T \oplus\left(B^{\prime} \cap G_{\alpha+1}\right)=G_{\alpha+1}$ and $j_{\alpha}\left(B^{\prime} \cap G_{\alpha}\right) \subseteq B^{\prime} \cap G_{\alpha+1}$. This implies that $j_{\alpha}(\phi(b), b)=j_{\alpha}\left(\phi_{\alpha}(b), b\right)=\left(\phi_{\alpha}(b)+\left(f_{\alpha}-\phi_{\alpha}\right)(b), b\right)$ must be $=(\phi(b), b)$, for all $b \in B$. This implies that $f_{\alpha}(b)=\phi(b)$ for all $b \in B_{\alpha}$ and so $\phi \mid B_{\alpha+1}: B_{\alpha+1} \rightarrow T$ is an extension of $f_{\alpha}: B_{\alpha} \rightarrow T$. This contradicts the choice of $f_{\alpha}$. Thus we conclude that $E$ cannot be a stationary set. Hence there is a closed and unbounded set $C$ in $\omega_{1}$ such that $E \cap C=\varnothing$. Then $B=\bigcup_{\alpha \in C} B_{\alpha}$ and, for each $\alpha \in C, B_{\alpha}$ is pure and decent in $B$. By Proposition 5, $B$ is a $B_{2}$-group.

In what follows, we continue to denote by $T$ the torsion group $T$ whose $p$-component $T_{p}$, for each prime $p$, is of the form $T_{p}=\oplus_{i<\omega} T_{p, i}$ where each $T_{p, i}$ is an unbounded direct sum of cyclic $p$-groups.

COROllary. Let $B$ be a torsion-free group of rank $\aleph_{1}$ in which each pure subgroup of finite rank is Butler. Assume $V=L$ holds. Then the following are equivalent:

(a) $\operatorname{Bext}(B, T)=0$.

(b) $\operatorname{Bext}(B, S)=0$ for every torsion group $S$.

(c) B satisfies the third axiom of countability with respect to decent subgroups.

(d) $B$ admits an $\omega_{1}$-filtration $B=\bigcup_{\alpha<\omega_{1}} B_{\alpha}$ such that for each $\alpha, B_{\alpha+1}=B_{\alpha}+C_{\alpha}$ with $B_{\alpha}$ pure, $C_{\alpha}$ a finite rank Butler group, and $B_{0}=0$.

PROOF. (c) $\Leftrightarrow$ (d) was proved in [1] while the proof of (d) $\Rightarrow$ (b) may be found in [4]. Now (b) $\Rightarrow$ (a) is trivial. One needs $V=L$ only in Theorem 8 to conclude that (a) $\Rightarrow$ (c). Observe that (c) is equivalent to $B$ having an $\omega_{1}$-filtration of decent subgroups.

Eklof [11] showed that Martin's Axiom (MA) implies that $\operatorname{Ext}(A, C)=0$ for any strongly $\omega_{1}$-free group $A$ of rank $\aleph_{1}$ and any countable group $C$. Since our torsion group $T$ is countable we obtain the following. 
TheOREM 9 (Meta THEOREM). Let $B$ be a torsion-free group in which every pure subgroup of finite rank is Butler. Then the implications $(\mathrm{a}) \Rightarrow(\mathrm{b})$ and $(\mathrm{a}) \Rightarrow(\mathrm{d})$ of the above corollary are undecidable in ZFC.

Proof. Let $A$ be a nonfree strongly $\omega_{1}$-free group. Then $A$ satisfies the hypothesis and, by Eklof [11], MA implies that $\operatorname{Bext}(A, T)=\operatorname{Ext}(A, T)=0$. Since the homogeneous groups of type $(0,0, \ldots)$ satisfying either (b) or (d) must be free [4] and since $A$ is not free, we conclude that $A$ satisfies neither (b) nor (d).

\section{REFERENCES}

1. U. Albrecht and P. Hill, Butler groups of infinite rank and Axiom 3, Preprint.

2. D. Arnold, Pure subgroups of finite rank completely decomposable groups, Proc. Abelian Group Theory Conference, Oberwolfach, Lecture Notes in Math., vol. 874, Springer-Verlag, 1981, pp. 1-31.

3. D. Arnold and C. Vinsonhaler, Pure subgroups of finite rank completely decomposable groups. II, Proc. Abelian Group Theory Conference, Honolulu, Lecture Notes in Math., vol. 1006, Springer-Verlag, 1983, pp. 97-143.

4. D. Arnold, Notes on Butler groups and balanced extensions, Preprint.

5. R. Baer, Abelian groups without elements of finite order, Duke Math J. 3 (1937), 68-122.

6. L. Bican, Splitting in abelian groups, Czechoslovak Math. J. 28 (1978), 356-364.

7. __ Purely finitely generated groups, Comment. Math. Univ. Carolin. 21 (1980), 209-218.

8. L. Bican and L. Salce, Infinite rank Butler groups, Proc. Abelian Group Theory Conference, Honolulu, Lecture Notes in Math., vol. 1006, Springer-Verlag, 1983, pp. 171-189.

9. L. Bican, L. Salce, and J. Stepan, A characterization of countable Butler groups, Preprint.

10. M. C. R. Butler, A class of torsion-free abelian groups of finite rank, Proc. London Math. Soc. 15 (1965), 680-698.

11. P. Eklof, Homological algebra and set theory, Trans. Amer. Math. Soc. 227 (1977), 207-225.

12. . Applications of logic to the problem of splitting abelian groups, Logic Colloquium 76 , North-Holland, 1977, pp. 287-299.

13. L. Fuchs, Infinite Abelian groups, vols. I and II, Academic Press, New York, 1971 and 1973.

14. P. Griffith, A solution to the splitting mixed group problem of Baer, Trans. Amer. Math. Soc. 139 (1969), 261-269.

15. R. Hunter, Balanced subgroups of abelian groups, Trans. Amer. Math. Soc. 215 (1976), 81-98.

16. R. Jensen, The fine structure of the constructible hierarchy, Ann. Math. Logic 4 (1972), 229-308.

Department of Mathematics, University of Colorado, Colorado Springs, Colorado 809337150 